\section{Die Arbeit mit Träumen bei Freud und heute}

Michael Ermann (Berlin)

Zusammenfassung: Freud konzipierte seine Traumdeutung nach dem Modell einer Neurose und sah in Ihnen das Ergebnis einer Verdrängung infantiler Wünsche. Seine Traumdeutung machte die verschleiernde Traumarbeit rückgängig und deckte den verdrängten Wunsch auf. Auf diese Weise wird die Kindheitsamnesie durch Erinnerung ersetzt und das Unbewusste vertrauter gemacht.

Diese Art der Traumdeutung ist für «klassische» Neurosen noch immer gültig. Daneben hat sich in den letzten Jahrzehnten das Konzept archaischer Träume Aufmerksamkeit verschafft, das vornehmlich bei den heute verbreiteten Persönlichkeitsstörungen Anwendung findet. Diese Träume unterscheiden sich wesentlich von den neurotischen Träumen. Sie stellen unverhüllt die Affektivität und das Befinden des Träumers dar und verweisen wie Zeichen auf den mentalen Zustand, in dem sie geträumt werden. Sie bedürfen keiner weiteren Interpretation. In der Behandlung fördern der Dialog und die Übersetzung der Zeichen in Sprache die Symbolisierung und helfen dem Träumer, sich zu begreifen, $d . h$. Begriffe für seine Zustände zu finden. Darüber hinaus macht er bei der therapeutischen Arbeit mit Träumen die Erfahrung, dass seine Zustände contained werden können.

Schlüsselwörter: Traum, Gedächtnis, Persönlichkeitsstörung, Behandlungstechnik

\section{Träume und der Wandel der Psychoanalyse}

Als Hintergrund für das erweiterteVerständnis der Bedeutung von Träumen und des Umgangs damit seien einige der Veränderungen markiert, welche die Psychoanalyse in den 120 Jahren seit ihrer Entwicklung durch Sigmund Freud um 1890 erfahren hat.

Freud befasste sich vornehmlich mit psychischen Störungen, die in einer relativ späten Phase der Entwicklung begründet sind, der von ihm so genannten ödipalen Phase, die wir in der Zeit nach dem Abschluss der basalen Strukturbildung um das 3. und 4. Lebensjahr ansiedeln. Diese Störungen bezeichnen wir heute als klassische Neurosen. Sie sind durch eine weitgehend ausgereifte Persönlichkeit gekennzeichnet und beruhen auf verdrängten Konflikten der Kindheitsentwicklung. Daneben haben wir es in der heutigen Praxis mit Persönlichkeitsstörungen zu tun, deren Kern in Entwicklungsstörungen begründet ist und bei denen ungünstige Entwicklungsbedingungen strukturelle Defizite und labile Ichstrukturen zurückgelassen haben. Freud und die frühe Psychoanalyse haben diese Patienten nich gesehen oder sie haben sie aus der Perspektive der klassischen Neurosen betrachtet und behandelt.

Dieser Wandel der psychotherapeutischen Klientel und die Entdeckung der frühen präödipalen Dimension psychischer Störungen haben eine Neukonzeption der therapeutischen Strategien nach sich gezogen, die heute in einer entwicklungsfördernden und strukturorientierten Behandlungstechnik seinen Niederschlag gefunden hat. Sie hat die ursprüngliche Konzeption der psychoanalytischen Therapie als konfliktorientierte Arbeit am Unbewussten in den Hintergrund treten lassen. Die Behandlungsstrategie bei Persönlichkeitsstörungen stellt die Transformation archaischer Ichzustände und die Neuerfahrung am anderen in das Zentrum der Behandlungsstrategie.

Mit dieser Umorientierung verbunden hat sich in der postfreudianischen Psychoanalyse die Einsicht durchgesetzt, dass der wahrscheinlich entscheidende Wirkfaktor der Psychoanalyse nicht, wie Freud glaubte, in der Rekonstruktion von Erinnerungen und Einsicht durch Deutung liegt, sondern in Beziehungserfahrungen während der Behandlung, welche alte Erfahrungen überschreiben Heute wissen wir, was Freud noch nicht wusste: Dass diese Beziehungserfahrungen auf einem vorsprachlichen Funktionsmodus beruhen und im archaischen prozeduralen Gedächtnis gespeichert sind, während die späteren Erfahrungen semantisch codiert sind und im später angelegten episodischen Gedächtnis gespeichert werden. Freuds psychoanalytische Kur zielte auf Veränderungen im episodischen Gedächtnis, während wir heute viel stärker die prozedurale Basis solcher Erfahrungen sehen.

Vor dem Hintergrund dieser Entwicklungen ergibt sich nun die Frage nach Veränderungen in unserem heutigen Verständnis von Träumen und für den Umgang damit in der Behandlung. Ich werde im Folgenden die Idee entwickeln, dass die klassische Traumdeutung, wie Freud sie konzipiert hatte, um eine entwicklungsfördernde Art der Traumbearbeitung erweitert worden ist, und dabei zwei Dimensionen des Träumens beschreiben (vgl. auch Ermann, 2005a): 
, Eine prozedurale Dimension: Sie nimmt auf frühe affektive und sensorische Beziehungserfahrungen Bezug, die aus dem implizit-prozeduralen Gedächtnis stammen und dort präverbal codiert sind. Bei diesem Modus in der Traumgestaltung spreche ich von archaischen Träumen. Sie entstehen in Zuständen tieferer Regression, wie wir sie insbesondere bei Persönlichkeitsstörungen kennen.

, Eine episodische Dimension:Sie beruht auf späteren, semantisch codierten Erfahrungen und Erinnerungen aus dem explizit-deklarativen Gedächtnis. Diese Art zu träumen prägt die Träume, mit denen Freud sich bei der Neurosenbehandlung beschäftigt hat. Man kann sie daher als neurotische Träume bezeichnen. Sie enthalten neben der episodischen immer auch eine archaische Dimension, welche die Traumepisoden als Stimmungen und Atmosphären oder Gefühlszustände begleitet.

\section{Freuds Traumdeutung}

Freuds Traumforschung basierte auf einer bestimmten Vorstellung des Wesens des Traumes und auf seinem Konzept der Traumdeutung (Freud, 1900). Dieses Konzept beruht auf einer Parallelsetzung zwischen Neurosen und Träumen, wie Freud sie gesehen hat. Beide beruhen nach seiner Auffassung auf der Verdrängung; der verdrängte psychische Inhalt bildet demnach den Kern der Neurosen und der Träume. Nach Freud handelt es sich dabei um infantile Triebwünsche oder um traumatische Erfahrungen einer Verführung in der Kindheit. Verdrängung ist also der Mechanismus sowohl der Neurosen - als auch der Traumentstehung.

Freud zeigte, wie es in der psychoanalytischen Kur gelingen kann, Verdrängungen aufzulösen und die kindliche Amnesie, die er als Kern der neurotischen Psychopathologie betrachtete, rückgängig zu machen. Bekanntlich war er bei seinen Behandlungen zuerst von der Hypnose ausgegangen. Als er sich jedoch damit auseinandersetzen musste, dass eine seiner Patientinnen, Elisabeth von R. nicht zu hypnotisieren war, wandte er einen Kunstgriff an, den er bei Hyppolyte Bernheim in Nancy gesehen hatte. Er legte der Patientin die Hand auf ihre Stirn und forderte sie auf, nun alles auszusprechen, was ihr durch den Sinn ging. So gelangte er zur freien Assoziation (Freud, 1895)

Als Material, zu dem er seine Patientinnen assoziieren liess, benutzte er besonders gern ihre Träume. In ihnen fand er verdrängte infantile Triebwünsche in einer verhüllten Form. Den Mechanismus der Verhüllung des ursprünglichen Traumgedankens, des verdrängten Triebwunsches, nannte er Traumarbeit.
Freud ging davon aus, dass Träume einen individuellen Sinn haben, den man durch Assoziationen erschliessen kann. ${ }^{1}$ Die Fremdhaftigkeit, die häufig unseren Träumen anhaftet, ist Folge der Entstellungen, die an ihrem ursprünglichen Sinn vorgenommen wurden. Mit der Technik der Assoziation gelangt man vom Traum, wie wir ihn nach dem Erwachen erinnern, zu seinem versteckten Sinn Freud (1900) spricht vom manifesten Trauminhalt und vom darin verborgenen latenten Traumgedanken.

Die latenten schlafstörenden Reize sind in der Regel unbewusste Wünsche, die von der Traumzensur nicht im Bewusstsein zugelassen werden. Die einzige Möglichkeit diese Traumzensur zu passieren ist die Entstellung. So entstehen die latenten Traumgedanken. Die Traumarbeit besorgt die dafür erforderliche Entstellung. Sie verdichtet mehrere Vorstellungen zu einer Einzigen oder verschiebt die Betonung von einem Motiv auf das andere. Dieser Prozess der Veränderung bewirkt, dass die manifeste Traumgeschichte nicht mehr als solche den eigentlichen Traumgedanken zum Ausdruck bringt, der den Träumer motiviert, sondern auf ein Drittes hinter dem Manifesten verweist. Diesen Ersatz für eine Vorstellung oder einen Begriff im Bewussten bezeichnet man als Symbolisierung (Freud, 1911; Jones, 1916). Wir können deshalb bei den neurotischen Träumen auch von Symbolträumen sprechen.

Die Hauptfunktion des Traumes ist nach Freud (1900) die eines «Hüters des Schlafs». Das geschieht einerseits durch die Verarbeitung von Tagesresten in Traumgeschichten, um das Erwachen zu verhindern. Freud führt als Beispiel dafür unter anderem Napoleon an, der das Geräusch einer explodierenden Höllenmaschine in einen Schlachtentraum umwandelte. Andererseits komm es im Traum zu einer Abfuhr unbewusster Wünsche in den Traumgeschichten. Wäre das nicht der Fall, so käme es zu einem ständigen Reiz, der keinen ruhigen Schlaf zuliesse. Der Traum, so verallgemeinerte Freud, dient der unbewussten Wunschbefriedigung.

Vor Freud hielt man Träume weitgehend für metaphysische Botschaften oder bedeutungslose Erregungen des schlafenden Seelenlebens. Deutungen beschränkten sich in der Regel auf Analogien nach dem Muster antiker oder mittelalterlicher Traumbücher. Mit seiner Traumlehre war Freud der Erste, der den Traum für eine sinnhafte Schöpfung des individuellen Seelenlebens hielt. Seine geniale Traumlehre hat für einen bedeutenden Teil der psychoanalytischen Behandlungen noch heute Gültigkeit - nämlich für die klassischen Neurosen, die nach Freuds Entdeckungen auf einer Verdrängungsabwehr beruhen. Sie werden heute auch als Konfliktstörungen bezeichnet. 
Traumdeutung heute: Zwei Modi des Träumens ${ }^{2}$

Heute beschäftigen wir uns in der psychoanalytischen Therapie aber nur noch zu einem begrenzten Anteil mit diesen höher strukturierten Neurosen. Ein grosser Teil unserer heutigen Klienten leidet unter Persönlichkeitsstörungen. In diesen Behandlungen spielt die Freudsche Symboldeutung von Träumen eine geringe Rolle, weil es sich um Patienten handelt, bei denen die Fähigkeit zur Symbolisierung noch nicht ausreichend entwickelt ist (vgl. Quindoz, 2016). Das Problem dieser Patienten sind weniger verdrängte Konflikte, die im Traum symbolisiert werden, als Entwicklungsstörungen, die sich u. a. in Symbolisierungsdefiziten niederschlagen. Bei ihnen geht es vornehmlich um Nachreifung und Neuerfahrung und um Zuwachs an basalen Ichfunktionen.

Wenn wir nach der «Traumdeutung heute» fragen, dann geht es aus meiner Sicht also darum zu klären, welche Struktur die Träume bei der Entwicklungspathologie dieser Patienten und Patientinnen haben und welche therapeutischen Konsequenzen sich daraus für den Umgang mit ihren archaischen Träumen ergeben.

Nach unserer heutigen Erfahrung steht die Traumgenerierung in einem engen Zusammenhang zum mentalen Funktionsmodus, in dem sich ein Träumer befindet (Lehmann \& Koukkou, 1983). Im implizit-prozeduralen Modus des Träumens entstehen archaische, niederstrukturierte Träume. Darin werden Traumbilder gestaltet, die wie Hinweisschilder auf basale affektive Zustände verweisen. Diese Träume werden von prozeduralen präsymbolischen Erfahrungen aus dem impliziten Gedächtnis geprägt. Sie werden auf geeignete Zeichen projiziert. Danach stehen die Traumbilder nicht für etwas anderes, sondern bringen direkt den affektiven Zustand zum Ausdruck, in dem der Traum geträumt wird. Hanna Segal $(1957,1991)$ spricht dabei von symbolischer Gleichsetzung. So entstehen konkretistische Zeichen- oder Zustandsträume, die unmittelbar darauf hinweisen, in welchem affektiven Zustand der Träumer sich befindet.

Dazu ein Beispiel, auf das ich später zurückkommen werde. Es handelt sich um einen Traum aus der Anfangsphase der Behandlung eines schwer regredierten Patienten. Er berichtete:

Ich bin in einer grossen, grell erleuchteten Halle, vielleicht ein Reaktor oder ein Kühlhaus. Ich suche etwas. Da sehe ich meinen Kopf in einem Glaskasten in der Ecke stehen. Es ist wie in einem Museum.
Im Gegensatz zu diesen archaischen Träumen entstehen die klassischen, «reifen» neurotischen Symbolträume im explizit-deklarativen Modus des Träumens. Sie sind aus Erinnerungen und semantisch codierten Erfahrungen gespeist, d. $h$. aus episodischen Gedächtnisinhalten. In diesem Modus werden Erfahrungen verträumt, indem aus diesem Material Traumgeschichten geformt werden, die in verschlüsselter Form den eigentlichen Traumgedanken enthalten. Der latente Traumgedanke kann ein Wunsch, eine Kränkung, ein Trauma oder vieles andere sein. Er wird im manifesten Traum verhüllt. Die Geschichten selbst sind Symbole für das verdrängte Erleben und bedürfen deshalb der Deutung, wenn man den ursprünglichen Traumgedanken erfassen will.

Als Beispiel dazu den Traum einer Patientin aus der Beendigungsphase ihrer Behandlung:

Ich treffe den Pfarrer und wundere mich, dass er grau geworden ist, ein gebrochener alter Mann. Er hält mir schweigend die Hand entgegen und sagt nur: «Mein Kind ... Ich will weggehen, aber da fällt mir ein: Ich habe ja noch nicht gebeichtet.

Massgeblich für die Traumgenerierung ist also der mentale Zustand, in dem der Träumer sich befindet (Lehmann \& Koukkou, 1983; Moser \& Zeppelin, 1996). Je nach Regressionsniveau wird dabei zwischen implizit-prozeduralen und dem explizit-deklarativen Modus unterschieden. ${ }^{3}$ Im impliziten Modus geht es um konkretistische Projektionen von affektiven Zuständen, im expliziten ausserdem um Verhüllung und Symbolbildung.

Zwischen dem archaischen und dem neurotischen Träumen bestehen fliessende Übergänge. Das liegt daran, dass der reifere implizit-episodische Funktionsmodus den früheren archaischen überlagert und ergänzt und nicht einfach ablöst und ersetzt. Das bedeutet, dass bei neurotischen Träumen imme auch die prozedurale archaische Dimension mitschwingt - z. B. als Atmosphäre oder als Stimmung im Traum. Dabei wird die archaische Dimension heute vie stärker gewichtet als früher.

Es gibt zudem einen Übergangsbereich, in dem beide Funktionsweisen sich abwechseln. Dieser Bereich tritt klinisch bei narzisstischen und depressiven Störungen in Erscheinung. ${ }^{4}$ Er ist durch ein Hin- und Herpendeln zwischen dem neurotischen und dem archaischen Träumen und durch die enge Verknüpfung zwischen beiden Modi des Träumens gekennzeichnet. Dazu später ein Beispiel. 


\section{Neurotisches Träumen: Freuds klassische Träume}

Wohlvertraut sind jedem Psychoanalytiker die neurotischen Träume wie sie von Freud konzeptualisiert worden sind. Ihre Botschaft lässt sich durch Desymbolisierung und Konstruktion des latenten Traumgedankens aus dem manifesten Text entschlüsseln. Dabei wird die ursprüngliche Traumarbeit, Freuds genialem Gedanken folgend, rückgängig gemacht.

Die Atmosphäre eines solchen Traumes soll am Beispiel der bereits oben erwähnten Patientin lebendig werden. Sie hatte bereits in der Anfangsphase ihrer Behandlung in der Übertragung ihre konflikthafte Vaterbeziehung aktiviert und träumte:

Ich gehe die Treppe zur Wallhalla ${ }^{5}$ hinauf, wo wir oft zum Schulausflug waren. Oben steht eine neue Statue. Ich denke, es ist König Ludwig, aber dann sehe ich überrascht, das ist mein Vater. Ein Kran beginnt, den riesigen Kopf abzuheben, so wie bei der Leninstatue in Berlin. ${ }^{6}$ Ich schreie noch, das geht doch nicht, aber niemand kann mich hören.

Welche Botschaft wollte dieser Traum vermitteln? Er zeigt der Patientin im König-Ludwig- bzw. Lenin-Motiv die anfängliche Idealisierung und beginnende Demontage ihres idealisierten Vaterbildes.

In der Schlussphase ihrer Behandlung träumte sie den bereits zitierten Traum vom Pfarrer:

(...) Ich wundere mich, er ist grau geworden, ein gebrochener alter Mann. Er hält mir schweigend die Hand entgegen und sagt nur: ‘Mein Kind ...〉

Dieser zweite Traum lässt erkennen, mit wie viel Schuldgefühl die Patientin ihre Loslösung und Verselbständigung verarbeitet hatte - ein Thema, das die Abschlussphase dieser Behandlung geprägt hatte.

Mehr als der Inhalt interessiert in unserem Zusammenhang die Form. Beide Träume berichten Geschichten, wie man sie sich erzählt. Beide enthalten Botschaften hinter dem erzählten Text, die sich durch Assoziation und Deutung erschliessen liessen und den Zugang zu bedeutenden Themen, gegenwärtigen Konflikten und ihren Kontexten in der Aktual- und Vergangenheitsperspektive eröffneten. Ganz im Sinne von Freuds Traummodell bilden sie neurotische Kompromisse zwischen unbewussten Gedanken und deren Abwehr - zwischen dem Wunsch nach Loslösung und den Schuldgefühlen. Es handelt sich um Beispiele für Träume, in denen auch die neurotische Übertragung ihren Weg über die Traumarbeit und die Traumzensur in die Behandlungsstunde findet.

\section{Träume als Ausdruck des Regressionsniveaus bei}

mittlerem Strukturniveau

Der folgende Traum stammt aus dem Beginn der Behandlung einer Patientin, die mit schweren Depressionen in die Analyse kam. Ihr Thema waren die Versorgung und die geringe Selbstfürsorge, die sie für sich aufbrachte. Diese Themen treten in der folgenden Traumgeschichte als verborgene Anspielungen in Erscheinung:

Ich bin in der Wohnung von Rosi [einer Freundin, ME] und will ihr Kind betreuen. Da merke ich, dass sie es drei Tage lang nicht gefüttert hatte. Ich bin völlig hilflos und weiss nicht, was ich tun soll - ich habe doch keine Kinder.

Sie fand sich spontan selbst in dem Kind wieder und erkannte zugleich ihre Identifizierung mit dem Bild einer (ihrer) Mutter, die nicht genügend gut für ihr Kind (für sie) sorgen konnte.

Es handelt sich nach meiner Erfahrung um den typischen Traum einer Frau mit einer narzisstisch-depressiven Persönlichkeit auf mittlerem strukturellem Entwicklungsniveau. Zu Beginn ihrer Analyse war sie in einem relativ gut integrierten Zustand. So zeigte ihr Traum zwar eine gewisse Ratlosigkeit, war im Übrigen aber kohärent und «erzählte» aus einer konstruktiven Distanz von den Empfindungen und Fantasien, die sie zu Beginn dieser Behandlung beschäftigten. Das alles kleidete sie in eine Traumgeschichte, in der Erzählung und Affektausdruck sich ergänzten.

Als sie in den darauffolgenden Wochen schwere Verletzungen in der Partnerschaft und berufliche Kränkungen erfuhr, geriet sie in eine tiefe Regression Es breitete sich eine Atmosphäre von Ratlosigkeit und Verzweiflung aus. So entstand der verständliche Wunsch, bei mir Schutz und Hilfe zu finden, was in konkrete Forderungen mündete, die mich überforderten. Damit ging ihre anfängliche Idealisierung unserer Beziehung und meiner Person verloren. Schliesslich begann sie, ihre aggressiven Affekte, ihre Wut und schliesslich ihren Hass auf mich zu pro- 
jizieren, wodurch ich ihr immer weniger hilfreich erschien. Sie wurde suizidal. In dieser Krise erzählte sie folgenden Traum:

Ich komme in einen Raum und finde dort einen bunten flauschigen Vogel, der schwer verletzt auf und ab flattert. Ich rede mit ihm wie mit einem guten Freund und nehme ihn in die Hand. Ich merke, er hat beide Beinchen gebrochen. Sie hängen kraftlos herunter. Das eine ist geschient, aber die Bandage, mit der die Schiene befestigt ist, ist locker. Ich denke, die Schiene wird ihm auch nicht mehr helfen.

In diesem Traum brachte sie ihre Verzweiflung unverhüllt zum Ausdruck Er unterscheidet sich in seiner Direktheit vom vorher berichteten Traum und bewirkte in mir eine Lähmung, wie ich sie selten erlebt habe. Ohne Einzelheiten zu wissen, erkennt man in diesem Traum einen Zustand von Hilflosigkeit und Hoffnungslosigkeit. Es geht hier um die Mitteilung und - wie man an meiner Reaktion sieht - um den Austausch von emotionalen Zuständen auf einer prozeduralen Ebene, auf der der Gefühlsausdruck eine bedeutend grössere Rolle spielt als kognitive Informationen.

Dieser Traum verweist auf ein verletzliches Selbst, das durch die geschilderten Umstände zutiefst erschüttert und labilisiert war. Man kann diesen Zustand auf eine Ich-Regression zurückführen. Sie hatte bewirkt, dass sie sich nicht mehr zu helfen wusste und diese Hilflosigkeit nun direkt und sehr konkretistisch in Forderungen und in archaische Träume umsetzte.

Im Verlauf der folgenden Monate gelang es, die Krise zu überwinden. Sie wagte einen Neuanfang in Beruf und Partnerschaft. Viel später träumte sie gegen Ende ihrer Analyse den folgenden Traum, der nun wieder deutlich die Merkmale und die Atmosphäre eines neurotischen Traumes aufwies:

Ich bin in Ihrer Wohnung, Sie sind irgendwo im Haus bei Ihrer Frau, die ist schwanger. Ich kann Sie also nicht sehen, weiss aber, dass Sie da sind. Ich liege gemütlich auf der Couch. Davor spielt Ihr Kind, ein kleines Mädchen mit weissblondem Haar, so wie ich als Kind war. Es stolpert, fällt und weint. Ich hebe es auf, tröste es und es lächelt mich an.

\section{Archaische Träume und niedrig strukturiertes Träumen}

Über niedrig strukturiertes Träumen gibt es vergleichsweise wenige Berichte. Das mag mit daran liegen, dass man mit Freud lange ein intaktes, also höher strukturiertes Ich als Basis für eine konstruktive Arbeit mit Träumen voraussetzte (z. B. Anna Freud, 1954) ${ }^{8}$.

Allerdings haben Analytiker, die sich mit der Behandlung von Psychosen befassten, schon immer auf die konkretistischen Inhalte niederstrukturierter Träume hingewiesen (Bion, 1957, 1962; Segal, 1957, 1991; Rosenfeld, 1965). Doch erst mit derVerbreitung der psychoanalytischen Borderline-Therapie in den 1970er Jahren fanden Träume von Patienten mit Persönlichkeitsstörungen ein breiteres Interesse.

Dabei rückte die Störung oder Einschränkung von Ichfunktionen wie Symbolisierung und Neutralisierung, also der diagnostische Aspekt von Träumen, in den Blickpunkt (Erikson, 1954; Blanck \& Blanck, 1979; Beese, 1983). Ungezügelte Aggressivität und Destruktivität bis hin zu Todesdrohung und Tötung, unverhüllte Sexualität, Zerstückelung und Zerfall des Körpers, Verwendung von Tieren oder leblosen Objekten zur Traumgestaltung, Entleerung und Verfolgung erscheinen dabei als Zeichen niederstrukturierter Träume. Ihnen allen ist das Bizarre und die Überfrachtung mit Affekt und Impulsivität eigen und dies gibt ihnen einen chaotischen Charakter. Damit verbunden ist ein völliges Fehlen von Scham- und Schuldgefühlen beim Bericht von derart bizarren Träumen mit erschreckendem Inhalt (Rohde-Dachser, 1983). So erzählte eine Patientin völlig ungerührt:

Ich habe geträumt, ich liege so da und irgendwie ist mein Brustkorb offen und niemand merkt es. Das stinkt entsetzlich. In meiner Hand fühle ich etwas Feuchtes. Als ich hinschaue, bemerke ich, dass ich mein Herz in der Hand habe. Ich versuche es in den Brustkorb zurückzuschieben, aber ich kriege es nicht mehr da hinein.

Die konkretistische, sehr unmittelbare Darstellung von erschütternden somatisch-affektiven Zuständen und die ungerührte Art des Berichtes verweisen auf einen typischen Borderline-Traum und das niedere Funktionsniveau. Solche Träume werden im Schlaf unter dem Eindruck von Erschütterungen im Wacherleben mit dem Material aus dem implizit-prozeduralen Gedächtnis geformt. Das bedeutet, dass die Bilder, die wir bei archaischen Träumen finden, nicht als Erinnerungen im Sinne erlebter Geschichten aufzufassen sind. Sie bringen nicht das Damals zum Ausdruck, sondern dienen dazu, den aktuellen inneren Zustand 
zu bezeichnen. Um sich auszudrücken, werden geeignete Bilder gewählt oder auch frei erfunden. Jedenfalls ist ihr Erinnerungsgehalt für das Verständnis nicht massgeblich. Entscheidend ist, welche Affekte, Impulse oder existenziellen Zustände durch bestimmte Bilder um Ausdruck ringen und nicht, welchen Erinnerungswert ein Motiv hat, das für die Projektion gewählt wird.

Das bedeutet, dass der Ansatz der klassischen analytischen Traumdeutung, der darauf abzielt, im Traum verborgene symbolisierte Bedürfnisse oder Beziehungsepisoden aufzudecken, bei niedrig strukturierten Träumen fehlschlägt. Damit wird der Unterschied zwischen neurotischen und archaischen Träumen noch einmal deutlich: Der niedrig strukturierte Traum ist ein projizierter IchZustand, eine Enthüllung, wie Kohut (1977) es genannt hat. Der neurotische Traum ist dagegen der symbolhafte Ausdruck eines verdrängten Motivs, ein Kompromiss zwischen einer verdrängten Erinnerung und ihrer Abwehr und insofern eine optimale Verhüllung.

\section{Vom Umgang mit archaischen Träumen}

Der Patient, dessen «Reaktortraum» ich anfangs geschildert habe, war im Zustand einer schweren Dekompensation mit Depressionen, Panik, Absencen und multiplen körperlichen Beschwerden in meine Behandlung gekommen. Es handelte sich um einen bis dahin erfolgreichen Geschäftsmann, der durch einen Betrug in kurzer Zeit sein gesamtes Vermögen verloren hatte, woraufhin auch seine Partnerschaft gescheitert war. Er stand jetzt, wie er einmal sagte, «nackt und bloss und völlig allein» da und fühlte sich plan- und kopflos. Für diesen Zustand hatte er in dem «Reaktortraum» Bilder gefunden, mit denen wir arbeiten konnten.

In der Beschreibung seines Selbst-Zustandes in diesem Traum bestand die bedeutende Aufforderung an den Analytiker, diesen Zustand zu teilen. Ich war erschrocken, aber es gelang mir, die Ruhe zu bewahren und nach einiger Zeit einen gewissen Abstand zurückzugewinnen. Dann konnten wir über die Traumbilder sprechen.

Er beschrieb zuerst die «unheimliche Stille» der Halle, die ihm Angst machte. Ich benannte diese Angst, die vor seiner völligen Selbstisolierung nur allzu offenkundig war. In der Helligkeit der Scheinwerfer fühlte er sich in einem Rampenlicht, in dem er nichts verbergen konnte. Ich sprach mit ihm über seine Scham angesichts des erlittenen Betrugs, der Einbusse seines Vermögens und des Verlustes seines sozialen Status. Er verachtete sich dafür. Er erkannte, dass ein Suizid, an den er immer wieder gedacht hatte, ihm als einfache Lösung seines Schamproblems erschienen war. Im Alleinsein erkannte er nun seinen selbst gewollten Rückzug, mit dem er sich vor der Scham schützte. Er begann zu überlegen, ob ihm sein Rückzug tatsächlich gut tat.

Dann sprachen wir über den Mann ohne Kopf. «Wie kann man ohne Kopf leben?», fragte er. Ich sagte: «Sie haben mir gesagt, dass Sie sich kopflos fühlen.» Schliesslich meinte er: «Es fühlt sich an, als ob ich Kopf und Verstand verloren habe.» Er überlegte, ob er seinen Kopf wohl vor seinen unermesslichen Aggressionen (dem Reaktor) in Sicherheit gebracht hatte. Ich äusserte meine Vermutung, dass die Aggression und Wut sich auch gegen die Betrüger richteten, denen er seinen Verlust anlastete. «Die wollten mich um Kopf und Kragen bringen», sagte er, und fügte schmunzelnd im Hinblick auf das Kühlhaus hinzu: «Da hilft es nur, cool zu bleiben.»

Über das Bild des Reaktors (Aufheizung) und des Kühlhauses (Unterkühlung) bekam er einen Zugang zu seiner mörderischen Wut. Ich wies ihn darauf hin, dass er in seiner Beschämung nur heiss oder kalt wahrnehmen konnte und die Möglichkeit nicht bemerkte, die im Reaktor gespeicherte Energie durch gesteuerte Nutzung für sein Leben zu verwerten. Das beeindruckte ihn sehr. Selbst das Kühlhaus bekam nun neben den Aspekten von Erstarrung und Kälte etwas Bewahrendes und erschien ihm nun auf Zukunft angelegt.

Dieser Traum steht an der Grenze zwischen niedrig strukturierter und neurotischer Traumtätigkeit. Er enthält nicht eigentlich Symbole sondern Zeichen, mit denen er seinen inneren Zustand markierte, doch das Nachsinnen darüber und die Selbstinterpretation, die der Patient nun zur Bewältigung einsetzte, zeigten, wie er zunehmend die Fähigkeit zurückgewann, über die Traumbilder nachzudenken und ihnen einen Sinn zu unterlegen. Meine Aufgabe fand ich darin, ihn dabei zu unterstützen und zu begleiten.

Diese Ausschnitte aus der Arbeit mit einem archaischen Traum, der zunächst wie ein einziger lautloser Schrei des Entsetzens erschienen war, zeigt bereits, wie die therapeutische Arbeit, die sich auf das Grauen des Traums einlässt, Bewältigungskräfte in einem solchen Traum-Ich-Zustand entdecken und freisetzen kann. Es handelt sich bei genauerer Betrachtung um einen verzweifelten, aber auch um einen kreativen Traum.

Als Psychoanalytiker findet man bei solchen Träumen seine Aufgabe darin, das Schöpferische für den Ich-Aufbau und die Progression zu nutzen, ohne dabe die Verzweiflung, Hoffnungslosigkeit, Destruktivität oder ein mögliches Scheitern zu verleugnen. Dabei haben Interventionen vor allem das Ziel, die Regression zu mässigen und den Dialog zu stärken. Sie sind mehr als supportive denn als interpretative Interventionen gemeint. Es geht darum, sich zur Verfügung zu stellen, 
um Projektionen von abgelehnten Affekten und Anteilen des Selbst aufzunehmen, auszuhalten und zu integrieren. Auf dieseWeise können solche Empfindungen und Fantasien geteilt, verändert und schliesslich in neuen Kontexten gesehen und erlebt werden. Behandlungstechnisch bedeutet das, gemeinsam über die Projektionen nachzusinnen und darüber in einen Dialog zu gelangen.

Welche positiven Entwicklungen sich dabei ergeben können, zeigt der folgende Traum dieses Patienten, den er ungefähr zwei Jahre später, gegen Ende seiner Behandlung, träumte: «Wir sind in einer Kaserne und werden von einem Monster verfolgt.» [In der Stunde wird klar, es sind er und ich als sein Analytiker, und die Kaserne ist die Klinik, in der seine Behandlung in meinem Arbeitszimmer im obersten Stockwerk stattfindet.] «Ich weiss, da gibt es ganz oben einen kleinen Raum, da sind wir sicher. Wir finden den Raum. Ich kann die Tür verschliessen.»

Wenn man diesen Traum mit dem «Reaktor-Traum» vergleicht, dann wird verständlich, was die Behandlung bewirkt hat. Zum einen erkennt man, dass eine abstrakte Traumszene einer belebten Traumgeschichte Platz gemacht hat. Damit reflektiert der Traum die Überwindung der regressiven Krise, in welcher der Patient zu mir gekommen war. Während ihn damals unerträgliche Ich-Zustände beherrscht hatten, für die der «Reaktortraum» ein Zeichen setzte, brachte er jetzt Beziehungsgeschichten zwischen uns in Traumbilder ein, in denen er zeigte, dass er begriffen hatte, wie er sich wieder schützen konnte.

Freud (1900) betrachtete die Konfliktanalyse von neurotischen Träumen als den Königsweg, der die Psychoanalyse in die Lage versetzt, die «Unterwelt», d.h. das Unbewusste zu bewegen. Im Laufe der Entwicklung der psychoanalytischen Behandlungstheorie und -technik hat der Traum diese Vorrangstellung an die Übertragung verloren. Demzufolge hat Greenson (1970) in seinen «psychoanalytischen Erkundungen» erneut für die Sonderstellung des Traumes in der psychoanalytischen Praxis geworben.

Das heutige Wissen über prozedurale Modi des Erlebens und der Erlebnisverarbeitung erschliesst auch der Arbeit mit Träumen eine neue Dimension jenseits der Konfliktanalyse: Die Dimension der basalen präverbalen Erfahrungen, die in allen Träumen mitschwingt. Bei Patienten mit Persönlichkeitsstörungen prägt sie das archaische Träumen. Indem die Erhellung der darin enthaltenen basalen Erlebniszustände in und jenseits der Übertragung in das Zentrum gerückt wird, erfährt die Arbeit mit Träumen auch bei Persönlichkeitsstörungen eine Neubewertung. Sie erhält die besondere Stellung zurück, die ihr - trotz der Konfliktorientierung im psychoanalytischen Mainstream - verloren gegangen war.

\section{Literatur}

Beese, F. (1983). Neuere Aspekte des Traums bei Übertragungsneurosen, narzisstischen Störungen und Psychosen. In M. Ermann (Hrsg.), Der Traum in der Psychoanalyse und analytischen Psychotherapie (S. 25-35). Berlin, Heidelberg \& New York: Springer.

Bion W. R. (1962/1990). Lernen durch Erfahrung. Frankfurt a. M.: Suhrkamp.

Bion, W.R. (1957/1990). Zur Unterscheidung von psychotischen und nicht-psychotischen Persönlichkeiten. In E. Bott Spillius (Hrsg.) (1988), Melanie Klein heute. Bd 1. (S. 75-99). Stuttgart: Klett-Cotta.

Blanck, G. \& Blanck, R. (1979/1980). Ich-Psychologie II. Stuttgart: Klett-Cotta.

Erikson, E. H. (1954). Das Traummuster der Psychoanalyse. Psyche-Zeitschrift für Psychoanalyse und ihre Anwendungen 10, 561-605.

Ermann, M. (2016). Psychotherapie und Psychosomatik. Stuttgart: Kohlhammer (6. Aufl.).

Ermann, M. (2005a). Träume und Träumen. Stuttgart: Kohlhammer (2. Aufl. 2014). Ermann, M. (2005b). Die Übertragung als Matrix der Traumgenerierung. Über höher- und niederstrukturierte Träume. Forum der Psychoanalyse $21,156-167$

Freud, A. (1954). Der wachsende Indikationsbereich der Psychoanalyse. In: A. Freud (1980), Die Schriften der Anna Freud, Bd. 5 (1349-1369). München: Kindler.

Freud, S. (1911). Brief an C. G. Jung vom 14. März 1911.

Freud, S. (1900). Die Traumdeutung. Gesammelte Werke (1966ff.) Band 2/3. Frankfurt a. M.: Fischer.

Freud, S. (1895a). Studien über Hysterie. Gesammelte Werke (1966ff.) Band 1 (S. 75-145). Frankfurt a. M.: Fischer

Greenson, R. (1970/1982). Die Sonderstellung des Traumes in der psychoanalytischen Praxis. In Greenson R., Psychoanalytische Erkundungen. Stuttgart: Klett-Cotta.

Jones, E. (1916). The theory of symbolism. British Journal of Psychology 9, 151-260. Kernberg, O. F. (1975/1978). Borderline-Störungen und pathologischer Narzissmus. Frankfurt a. M.: Suhrkamp.

Kohut, H. (1977/ 1979). Die Heilung des Selbst. Frankfurt a. M.: Suhrkamp.

Lehmann, D. \& Koukkou, M. (1983). Psychophysiologie des Traums. In M. Ermann (Hrsg.), Der Traum in Psychoanalyse und analytischer Psychotherapie (S. 54-67). Berlin, Heidelberg \& New York: Springer.

Markowitsch, J. (2002). Dem Gedächtnis auf der Spur. Vom Erinnern und Vergessen Darmstadt: Wissenschaftliche Buchgesellschaft (3. Aufl. 2009). 
Moser, U. \& v. Zeppelin, I. (1996). Der geträumte Traum. Stuttgart: Kohlhammer.

Quindoz, J. M. (2016). Von der symbolischen Gleichsetzung zur symbolischen Repräsentation in Träumen. Forum der Psychoanalyse 32, 233-244.

Rohde-Dachser, C. (1983). Träume in der Behandlung von Patienten mit schweren Ich-Störungen. In M. Ermann (Hrsg.), Der Traum in der Psychoanalyse und analytischen Psychotherapie (S. 106-119). Berlin, Heidelberg \& New York: Springer.

Rosenfeld, H. (1965/1981). Zur Psychoanalyse psychotischer Zustände. Frankfurt a. M.: Suhrkamp.

Segal, H. (1991/1996). Traum, Phantasie und Kunst. Stuttgart: Klett-Cotta.

Segal, H. (1957/ 1990). Bemerkungen zur Symbolbildung. In E. Bott Spillius (Hrsg.) (1988/1990), Melanie Klein heute. Bd 1 (S. 202-224). Stuttgart: Klett-Cotta.

\section{Anmerkungen}

1 Dabei handelt es sich übrigens nicht um wirklich «freie» Assoziationen, sondern Freud liess seine Patientinnen gezielt (angeleitet) zu einzelnen Traummotiven assoziieren.

2 Die folgenden Passagen sind eine Neufassung eines Textes, der zuerst im Forum der Psychoanalyse 21 (2005b)156-167 erschienen ist.

3 Zum Stand der Gedächtnisforschung vgl. Markowitsch (2002 / 3. Aufl. 2009).

$4 \quad$ Als sog. mittleres Strukturniveau. Zur psychoanalytischen Strukturdiagnostik vgl. Ermann (2016).

5 Ruhmeshalle auf einer Anhöhe über der Donau.

6 Im Film «Good bye Lenin» (2001/2003) von Wolfgang Becker wird der politische und ökonomische Umbruch nach der deutschen Wiedervereinigung durch den Abbau der Lenin-Statue in Ostberlin markiert.

7 Auf die Übertragungsimplikationen dieser Träume wird hier nicht eingegangen.

8 «Wo diese Voraussetzung nicht gegeben ist, entstehen technische Schwierigkeiten ... [Dann] sieht der Analytiker sich gezwungen, die analytische Einsicht und Technik durch andere Beobachtungsformen zu ergänzen» (Anna Freud 1954).

\section{Angaben zum Autor}

Michael Ermann, Univ.-Prof. emeritus, Dr. med. habil., Facharzt und Psychoanalytiker, lebt in Berlin. Mitglied und Lehranalytiker der DPG, IPA, IFPS und der Akademie für Psychoanalyse München. 1979 Habilitation für Psychoanalyse und Psychosomatik an der Universität Heideberg. 1985 bis 2009 Abt.-Vorstand für Psychosomatische Medizin und Psychotherapie an der Ludwig-MaximiliansUniversität München. Zahlreiche Publikationen über Träume: 2005 Träume und Träumen, Kohlhammer Stuttgart (2. Aufl. 2014). Weitere Informationen unter: www.m-ermann.de. 